\title{
A theory-driven reflection on context-aware support for collaborative discussions in light of analytics, affordances, and platforms
}

\author{
Carolyn Rosé $^{1} \cdot$ Sanna Järvelä
}

Published online: 13 January 2021

(c) International Society of the Learning Sciences, Inc. 2021

\section{Introduction}

One of the overarching aims of all scientific communities is to facilitate the accumulation of knowledge as core members of those communities seek to contribute novel insights and new knowledge as a result of their participation within this joint venture. In light of that aim, and as the year of 2021 draws to a close, we offer this reflective issue of the International Journal of Computer-Supported Collaborative Learning (ijCSCL), inspired by an EARLI 2021 symposium considering collaborative dialogue through a weaving together of multiple theoretical perspectives. The symposium was organized by Michael Baker and Baruch Schwarz, with our former Editor-in-Chief, Sten Ludvigsen, as discussant, and was in turn partly prompted by synergistic thinking from an editorial from the September issue of ijCSCL by Peter Reimann (2021). A narrative recounting perspectives from that symposium, authored by the co-organizers and discussant, appears as the final contribution of this issue, published here as a Squib. We challenge our readers to read the four full articles of this issue, positioned ahead of the Squib in the line up, in light of the reflection, which will bring us full circle.

The challenge that prompted the symposium discussion is the changing reality of students coming to "the CSCL classroom". The argument is that the nature of the dialogue students more typically engage in within the environments of their current reality, in particular, with their native Internet and social media practices, are in flux. Their practices pose new challenges as these environments are associated with different "knowledge structures and knowledge taxonomies" (Crook, 2012) than environments that have housed more typical classroom studies, even with very large data sets, until now. The most important point of the Squib is the challenge to think bigger, to realize that conversations transcend the boundaries of activities within courses, extend over long periods of time, and comprise

Carolyn Rosé

cp3a@andrew.cmu.edu

Sanna Järvelä

sanna.jarvela@oulu.fi

1 Carnegie Mellon University Language Technologies Institute and HCI Institute, Pittsburgh, PA, USA

2 University of Oulu, Oulu, Finland 
an intricate interplay between levels. Only in establishing a bird's eye view that enables visualization and understanding of the connections between levels can we really understand the communicative and collaborative processes occurring at the societal level (Uttamchandani et al., 2020), which are especially consequential in light of recent historical events. The four full articles of this issue each present studies, mainly of a more typical scale, largely housed in typical kinds of environments studied previously. We therefore challenge our readers to consider how findings from these traditional environments bridge into the forward-looking perspective offered by the Squib, prompted by questions raised in its reading.

In particular, we begin with two articles contributing new methodological approaches in the area of learning analytics applied to discussion data. Next, we reflect on discussion from the perspective of affordances with an article that explores how platform affordances related to mediation of group interaction affect the nature and dynamics of that conversational interaction. Finally, we offer the final full article, which introduces a new collaborative platform, an environment built to facilitate collaborative software development. We close with some final reflections on the year of 2021.

\section{Analytics}

As we reflect on our conceptualization of collaborative discussion at different time scales, we are also faced with the issue of scale. As a community, we value both deep and thick descriptions of small portions of data as well as technology supported analyses of massive amounts of data, and everywhere in between along this spectrum (Hmelo-Silver \& Jeong, 2021). The field has witnessed the emergence of collaborative analytics over the past decade and a half, which enables gaining a bird's eye perspective over massive amounts of data, though ample opportunities for improvement of these technologies are still in abundance. We offer here two contributions to expand this important area.

First, Mohammed Saqr and Sonsoles López-Pernas have contributed an article entitled "Modelling diffusion in computer-supported collaborative learning: A large scale learning analytics study". This study employs a network analytic approach to investigation of role taking in large scale collaboration. While the learning under investigation occurs within a traditional learning environment, what is striking is that the analysis is based thousands of students and nearly a hundred thoughts total interactions, thousands of which were hand coded. The analysis employs a particular analytic construct, namely diffusion-based centralities, which were demonstrated to elucidate processes of information exchange and uptake within a broadscale collaboration. Validations were conducted utilizing comparison with measures of academic achievement and qualitative analysis of interactions. The analysis serves as a proof-of-concept for using such analytics to gain a vantage point that enables investigations of collaborative processes in large scale data beyond an individual randomized control trial.

Next we have an article by Jauwairia Nasir, Aditi Kothiyal, Barbara Bruno, and Pierre Dillenbourg entitled "Many Are The Ways to Learn-Identifying multi-modal behavioral profiles of collaborative learning in constructivist activities". A key aspect of this work is that it features an integration of data across multiple modalities including video, audio and $\log$ data in order to identify multi-modal collaborative learning behavioral profiles for dyads working on an open-ended task around an interactive tabletop with a robot as a mediator. The different modalities can be expected to provide complementary views on a complex, multi-faceted interaction in a physical space. In order to focus the interaction, 
the driving question is related to distinguishing behavior patterns of groups that learn from those that do not. Not only actions but also affect are considered together, with affect offering a means for explaining the connection between behavior and learning. Meaningful names are attributed to behavior patterns in order to capture the gestalt essence of the experience of groups within each of three clusters: namely, Expressive Explorers, Calm Tinkerers, and Silent Wanderers. The important point is that it is not the individual behaviors themselves that are valuable or not for learning. Emergent processes signify the existence of underlying intents, strategies and emergent processes, which on the one hand might be harder to pinpoint, but on the other hand are more explanatory.

\section{Affordances}

Real time analytics enable context sensitive triggering of support for collaborative learning. In order to use analytics purposefully to this end, we must understand how to create affordances that shape interactions in desired ways (Kreijns et al., 2002). Thus, research studying affordances for collaboration are critical.

To this end, Cixiao Wang and Shuling Li have authored "The trade-off between individuals and groups: Role interaction under different technology affordance conditions". As the name suggests, an experimental manipulation is investigated, which provides a landscape in which to view and discussion behavioral patterns. In this case, the manipulation takes place in part by means of a device-to-student ratio. In other words, collaborative processes emerge differently when students each have their own device, which might be assumed to be the ideal case, than when devices are shared. Another means for manipulating affordances for interaction is through the more typical means of a script.

A multifaceted approach was taken in the analysis, including thematic analysis, descriptive statistics, and social network analysis. Several dimensions of role-taking were investigated, including role emergence, role coordination, and group structure. Interpretation of this analysis enabled viewing the collaboration from the perspective of social status as teams worked together to establish norms and collective consciousness. An interesting dichotomy between efficient collaboration on the one side, which is valued in terms of outcomes, and internal coordination and close interaction on the other side, which is valued from the perspective of collaboration quality.

\section{Platforms}

The impact of collaborative learning technologies occurs as learners come together to experience supported activities on collaborative platforms. In comparison with the number of individual learning platforms in existence, collaborative platforms are still the minority. Though we do not advocate for making all learning experiences collaborative, it is worth noting that many environments for individual learning could potentially be extended to afford collaborative learning. The final full paper of this issue is one in which Ting-Chia Hsu, Hal Abelson, Evan Patton, Shih-Chu Chen and Hsuan-Ning Chang study such a collaborative environment, built as an extension of an established environment for individual learning and exploration. In particular, that article, entitled "Self-Efficacy and Behavior Patterns of Learners Using a Real-Time Collaboration System Developed for Group Programming", presents and evaluates a collaborative version of the well known MIT App 
Inventor (MAI) environment. Analytics are applied in order to gain visibility into the patterns found within the data.

MIT App Inventor (MAI) is popular block-based programming (BBP) learning environment designed to aid new programmers in making quick progress towards producing computer programs by removing the need for as much knowledge of programming language syntax. This study offers the opportunity to explore the types of interactions afforded by a platform that was not originally designed to be collaborative. Three dimensions of contrasts provide a lens for viewing the afforded types of interactions. First, behavior patterns towards problem solving are compared between individual and collaborative work as participants experienced the platform in both of these modes. Collaborative interaction patterns are compared between individuals with a computer science background with those from other disciplinary backgrounds as teams were formed as heterogeneous or homogeneous in terms of disciplinary background. Teams are contrasted with respect to their level of self-efficacy and the nature of their interaction patterns. In particular, a concern might be that participants with a non-CS background might have lower self-efficacy on the platform, but the evidence of this did not emerge. Significant interactions patterns were found signifying coordination of behavior between participants on teams, suggesting that the environment does promote collaboration.

An important characteristic of behavior in collaborative settings was the extent to which the behaviors of multiple students were coordinated. Beyond the results of the specific study featured in the paper, the analysis serves as a proof-of-concept that analytics can be used on this platform to identify collaborative behaviors of interest. This proof-of-concept to that effect paves the way for broader investigation of collaboration on this platform over longer periods of time in order to investigate collaboration that occurs over much longer times scales.

\section{Theoretical reflections}

The final contribution of the December issue is a Squib that brings us full circle with a theoretical reflection from Michael J. Baker, Baruch B. Schwarz, and Sten R. Ludvigsen entitled "Educational dialogues and Computer-Supported Collaborative Learning: critical analysis and research perspectives". The aim of the reflection presented in this Squib is to argue for the necessity for a deep, theoretical understanding of discussion and its unit of analysis in order to inform development of methods for analyzing discussion over different timescales. The challenge is to view collaboration at a societal level over long periods of time. Adopting such a lens is critical if we are to apply what we know about collaboration learned from small scale studies to large scale societal challenges where the insights are most needed. For example, we have witnessed in the past two years a worldwide argumentative knowledge construction of conceptions of COVID and problem solving regarding how to stay safe and healthy while sustaining other dimensions of life beyond physical health. Within this we see real differences in perspective, identity-based clashes of practices, and struggles to understand something no one yet fully understands. This collaborative knowledge construction is occurring around us in an immense group over a very long period of time within an ill-defined activity. Where are the interactions? They are everywhere and nowhere, occurring simultaneously, including interactions that are more direct with interactions that occur underneath the surface or behind the scenes, crossing modalities through multivocal interconnectedness. If we can understand, might we offer support? 
Their reflection proceeds in three parts, focusing first on collective thinking and its enactment and expression through dialogue, then on critical considerations regarding timescales and finally how they are accounted for in units of analysis applied to platform data. In the style of a richly textured tapestry woven from distinct threads of very different natures, the article integrates across a number of different theoretical perspectives, each contributing some insights about the nature of collaboration, as it may occur and be studied from the vantage point of multiple time scales. Depending upon the time scale and what is treated as the basic unit of interaction, questions are raised that are meant to prompt the kind of reflection that might enable bridging from basic science into the everyday world around us.

Finally, the Baker et al. Squib reminds us how deep theoretical tradition computersupported collaborative learning research has been built. Ultimately, CSCL is for humans, their interactions and dialogue in local and global contexts. While powerful new thick and big data and automatic analyses progress the field in terms of accumulating evidence of types and nature of interactions, general theories of dialogue, cognition and collective activity guide our chosen units of analysis, and help explain their deep meaning.

\section{1: Closing out the year}

In this final issue of 2021, we offer our thanks to this community for its commitment to the journal since its inception. We are proud of its growth, international representation, and healthy impact factor.

Weare now halfway through our four year term as co-editors-in-chief. We thank our authors, editorial board, and ad hoc reviewers, without whom none of this would be possible. We know it has not been easy for anyone to keep up with reviewing and revisions with the world as it has been. We have worked with great determination to keep the journal strong in the midst of all of this.

We would like to challenge the field to stay in the forefront and keep working hard with the important question raised by Baker et al.: "how can such diverse data sets, over multiple timescales, involving a combination of automatic and human analyses, be combined in a meaningful way ". For the next year's submissions we especially welcome "dialogue" in between data and theories.

We look forward to the coming year with great hope for better days ahead. On the horizon is the in preparation special issue, co-edited by Freydis Vogel and Lenka Schnaubert, which is slated to come out in 2022. We are excited to see that taking shape, and to have launched the second open call for special issue proposals, with a proposal submission deadline in early Spring.

\section{References}

Crook, C. (2012). The 'digital native' in context: tensions associated with importing Web 2.0 practices into the school setting. Oxford Review of Education, 38(1), 63-80. https://doi.org/10.1080/03054985.2011. 577946

Hmelo-Silver C. E., Jeong H. (2021). An Overview of CSCL Methods. In: Cress U., Rosé C., Wise A. F., Oshima J. (Eds.), International handbook of computer-supported collaborative learning. Computer-Supported Collaborative Learning Series, (vol. 19). Springer. https://doi.org/10.1007/ 978-3-030-65291-3_4 
Kreijns, K., Kirschner, P. A., \& Jochems, W. (2002). The sociability of computer-supported collaborative learning environments. Journal of Education Technology \& Society, 5(1), 8-22.

Reimann, P. (2021). Socio-technical configurations for productive talk. International Journal of ComputerSupported Collaborative Learning, 16, 301-306. https://doi.org/10.1007/s11412-021-09354

Uttamchandani, S., Bhimdiwala, A., \& Hmelo-Silver, C. E. (2020). Finding a place for equity in CSCL: Ambitious learning practices as a lever for sustained educational change. International Journal of Computer-Supported Collaborative Learning, 15, 373-382. https://doi.org/10.1007/s11412-020-09325-3

Publisher's note Springer Nature remains neutral with regard to jurisdictional claims in published maps and institutional affiliations. 